\title{
Optimal Triangulation by Means of Evolutionary Algorithms
}

\author{
Giovanni Prestifilippo \\ Dept. of Computer Science \\ University of Dortmund \\ Germany \\ giovanni@iml.fhg.de
}

\section{Introduction}

The reconstruction of surfaces from measured contour points is an obviously difficult task. The most common technique in this field is to approach the original surface by triangulation of the given points. A mesh of triangles is constructed such that each point belongs to at least one triangle. The surface obtained by triangulation can now be further improved by smoothing and ray tracing.
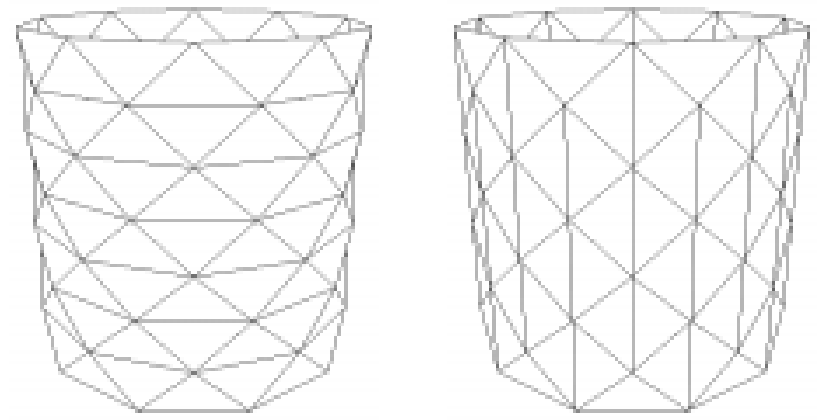

Fig. 1: Triangulations of a cylinder: Deterministic using the maxmin-criterion (left), probabilistic using the total absolute curvature.

Usually, three-dimensional objects cannot be scanned in one pass, but must be measured in a series of linear scans along parallel scan lines. Simple triangulation methods make use of the line structure of this kind of scans by keeping the scan lines fixed and calculating zig-zag paths between each pair of adjacent scan lines. The success of those strategies highly depends on the appropriate direction of the scan lines. Fig. 1 shows two triangulations of a cylinder with horizontal scan lines. While the left trian-

\author{
Joachim Sprave \\ Dept. of Computer Science \\ University of Dortmund \\ Germany \\ joe@LS11.informatik.uni-dortmund.de
}

gulation follows the scan lines, the right one was allowed to choose arbitrary triangles. The resulting surfaces (fig. 2) show how important the direction of the scan lines is.
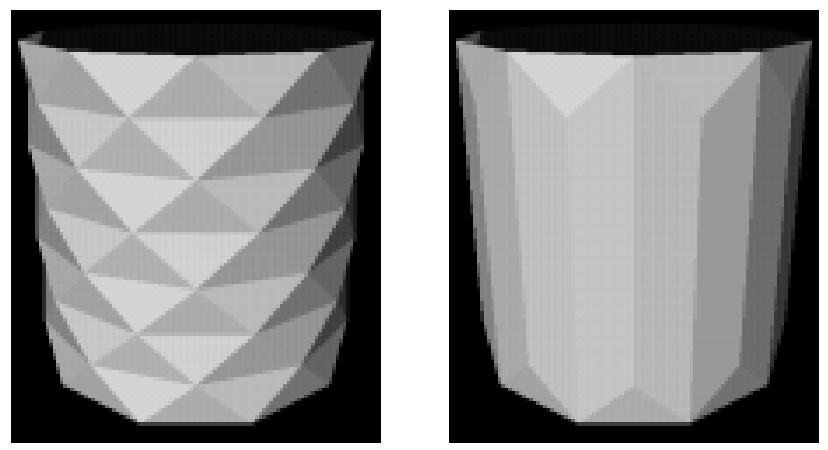

Fig. 2: Resulting surfaces from the triangulations in fig. 1.

\section{Quality Criteria}

In order to treat triangulation as an optimization problem, it is necessary to define what a good triangulation of a given set of points is. Although it is not difficult to compare two triangulations of the same object by simply looking at their ray-traced pictures, it is a non-trivial task to formally define a quality criterion.

Triangle based criteria start with a local measure that defines the quality of a single triangle, e.g. the smallest angle of the triangle. This leads to the widely used max-min angle criterion: Let be $T$ and $T^{\prime}$ two triangulations of the same set of points, and be $\tau_{i}$ and $\tau_{i}^{\prime}, i=1, \ldots, l$, lists of the triangles from $T$ and $T^{\prime}$. Let be $\min \phi_{i}$ the smallest angle in $\tau_{i}$ and $\min \phi_{i}^{\prime}$ the smallest angle in $\tau_{i}^{\prime}$, then a 
better-than-relation $\triangleleft$ on triangulations can be defined as

$$
T \unlhd T^{\prime} \Leftrightarrow \quad \sum_{i=1}^{l} \min \phi_{i} \geq \sum_{i=1}^{l} \min \phi_{i}^{\prime}
$$

The corresponding optimization problem can now be written as follows:

$$
f(T):=\sum_{i=1}^{l} \min \phi_{i} \rightarrow \max !
$$

In recent works[3,8], curvature based criteria were applied to the surface reconstruction problem with some success. In contrast to triangle based criteria, curvature based criteria do not only aggregate properties of single triangles. Instead, they try to achieve small angles between the normals of at least two adjacent triangles. For the work described in this paper, a relatively new curvature based criterion, the total absolute curvature criterion (TAC) was used. For a complete definition, the reader is referred to the original work[1]. In the following, a simplified definition is given which does not cover all possible situations.

For each inner point $P$ of a triangulation, the local curvature is measured by the absolute difference of $2 \pi$ and the the sum of all angles starting in $P$ : Let be $\tau_{i}, i=1, \ldots, d$ the $d$ triangles sharing $P, \phi_{i}^{P}$ the angle of $\tau_{i}$ starting in $P$, then the local TAC of $P, \operatorname{tac}(P)$ is defined as

$$
\operatorname{tac}(P):=\left|2 \pi \Leftrightarrow \sum_{i=1}^{d} \phi_{i}^{P}\right|
$$

If this value is zero, the angles in $P$ add to a full circle, so all triangles sharing point $P$ are coplanar. The TAC for an entire triangulation $T$ with inner points $P_{i}, i=1, \ldots, n$ is defined as the sum of the local TACs:

$$
T A C(T):=\sum_{i=1}^{n} \operatorname{tac}\left(P_{i}\right) \rightarrow \min !
$$

\section{Reference Algorithms}

Triangulation is a combinatorial problem. Since the search space increases exponentially with the number of points, total enumeration is impossible for real applications. Instead, most deterministic algorithms use certain heuristics in order to improve a given initial triangulation. Consequently, the result does not only depend on the strategy itself, but also on the starting configuration. Initial triangulations are usually obtained by a Delaunay triangulation, a simple scan line based algorithm which prefers short diagonals. As shown in fig. 1 and 2, this results in very low quality in the worst case.

For the comparison described in this paper, a new approach based on Dijkstra's shortest path algorithm was used. In contrast to traditional algorithms, it does not calculate an aggregation of local measures, but provides a single quality measure for all triangles between two scan lines. As shown in the original work, this results in a significant improvement over the widely used Delaunay based algorithms.

The only non-deterministic algorithm which has been applied to triangulation is Simulated Annealing (SA) [4]. The implementation used by the authors follows mostly the work of Schumaker[7]. The only modification is the use of TAC as quality measure instead of triangle based criteria.

\section{Evolutionary Approach}

The algorithm presented here bases on Evolutionary Algorithms as a generalization of the basic principles which were developed in the $60 \mathrm{~s}$ and $70 \mathrm{~s}$. For an overview of the paradigms, see e.g. the introducing chapters of [2].

\section{Internal Representation}

Since we already have vertices and edges, surface reconstruction can be easily modeled as a graph problem. Examples for appropriate operators can be found in literature, e.g. [5]. Since every triangulation for a given set of points consists of the same number of edges (and triangles, respectively), this representation would not only allow to code each valid triangulation, but also a huge number of invalid graphs. Since there is no simple representation that codes exactly the domain of valid triangulations, the way out of this dilemma is to define operators which guarantee to stay in the domain of valid triangulations. Furthermore, the first generation must be initialized by an algorithm that generates valid configurations. 


\section{Mutation}

A very natural variation operator is the edge swap operator which is also used in SA algorithms. In a two-dimensional projection, each inner edge defines a trapezoid which is obtained by removing the edge. If the trapezoid is convex a new edge can be inserted connecting the two other points in the trapezoid, as sketched in fig. 3. Before the mutation operator alters an edge, it checks whether it is swappable or not.

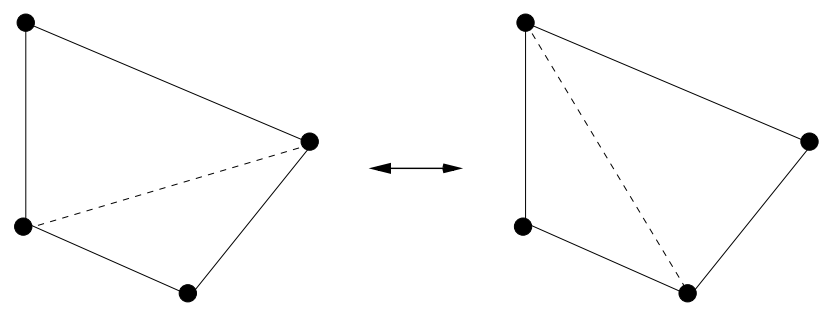

Fig. 3: The edge swap operator (schematically).

While in the SA a fixed number of edges is swapped in each iteration, the EA operator swaps each swappable edge with a certain probability. This mutation rate is fixed for an entire experiment.

\section{Recombination}

The natural way to create new triangulations from two parents is to cut both parents into two pieces along the same trajectory, and to perform crossover on the pieces. Since the cut removes edges, a repair operator must be applied to replace the lost edges. In [6], a simple deterministic triangulation strategy was applied to connect the pieces along the cutting line.

The problems of repair operators are obvious: Either they are good optimizers by themselves, then it is possible that an EA works even without selection, or they introduce a huge amount of random changes. In the latter case, the quality of recombined individuals is likely to be very poor.

\section{Selection}

The selection operators of the EA triangulation are taken from the Evolution Strategies. The $(\mu+\lambda)$ strategy produces $\lambda$ children from $\mu$ parents, and selects the best $\mu$ of the $\mu+\lambda$ individuals. Hence, only improvements are accepted. The $(\mu, \lambda)$ selection discards the parents before selecting, thus allowing deterioration. Therefore, this method requires to generate a surplus of children, i.e. $\lambda>$ $\mu$.

\section{Results}

Starting with simple geometric forms, like the cylinder in fig. 1 and 2, numerous experiments were made to find out whether EAs are appropriate methods for surface reconstruction. While the EA behaved very robust against variation of the selection pressure and mutation rate, the crossover operator did not work as expected. Since the results with recombination were always worse that without, all experiments described in the following were made with recombination switched off.

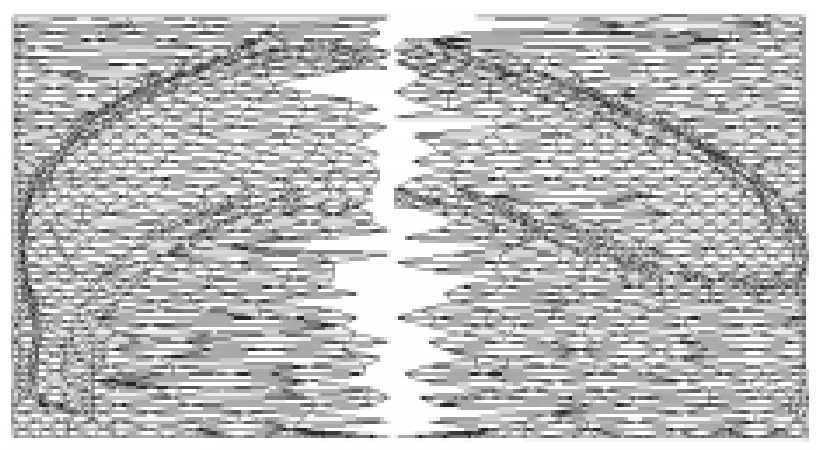

Fig. 4: A typical crossover cut.

The destructive effect of the crossover operator is shown in fig. 4 and 5. The scanned contour of a banana was optimized for some generations, then cut vertically (fig. 4). Figure 5 shows the result after applying the repair operator.

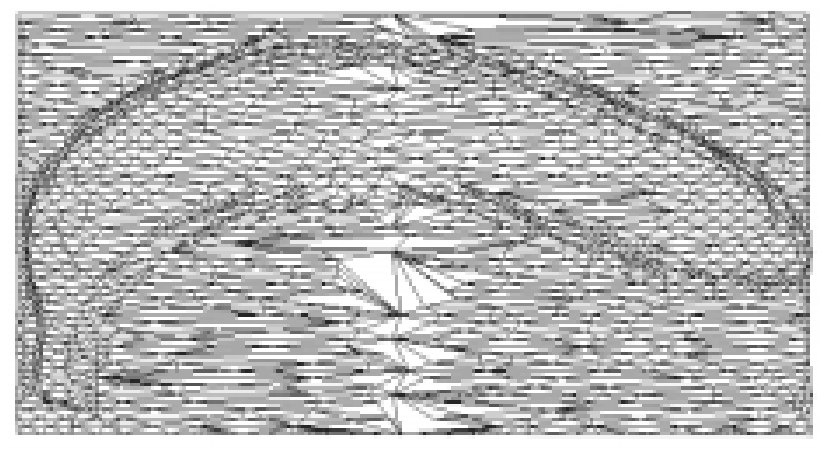

Fig. 5: An example of the effect of the repair operator.

In comparison to deterministic algorithms, the EA always produced the better results, especially when there are curvatures in different directions, which can be assumed for 
every real world application. Of course, the EA requires much more computation time, which may be a problem in real time applications.

Compared to the SA, the EA needs more time to produce results of the same quality. A reason for this may be the fixed mutation rates. In fact, first experiments (see [6] for details) with the mutation rate decreasing over the time showed a significant speed up of the EA. Since a fixed external schedule introduces a new external parameter that needs to be tuned, self-learning of mutation rates, as investigated in [2] seem to be a reasonable extension.

Both, SA and EA, do not depend on the quality of the initial configuration, even when started with a triangulation pessimized by a deterministic algorithm with inverted quality criterion.

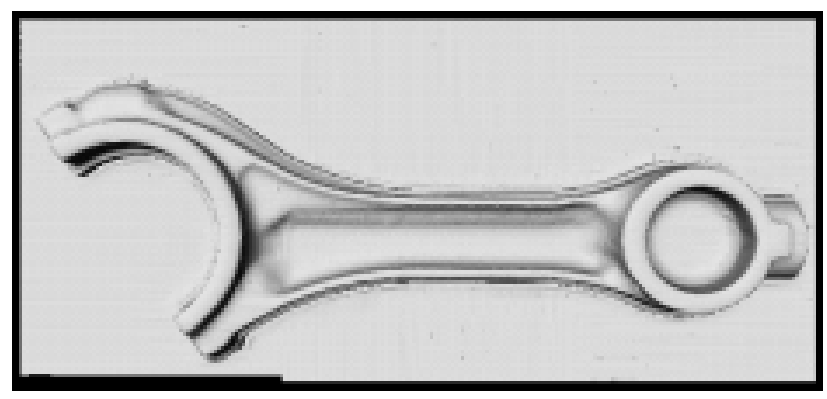

Fig. 6: The surface of the connecting rod used for comparison.

The example triangulation problem presented here is a connecting rod. Fig. 6 shows the entire shape. It can be seen that the left third of the connecting rod was scanned separately with the scan lines directed from left to right, while the rest was scanned from top to bottom, in order to provide appropriate scan lines for deterministic algorithms. Fig. 7 shows the triangulation of the left part obtained by the shortest path algorithm. The structure is very regular, and small triangles are preferred. In comparison, the EA is able to freely arrange the triangles without being bound to the scan lines (fig. 8). Therefore, curvatures in arbitrary directions can be approximated by properly aligned triangles. Although the deterministic solution looks smoother in the grid representation due to its regular structure, the reconstructed surfaces (fig. 9 and 10) show that the EA has undoubtedly produced a better solution.

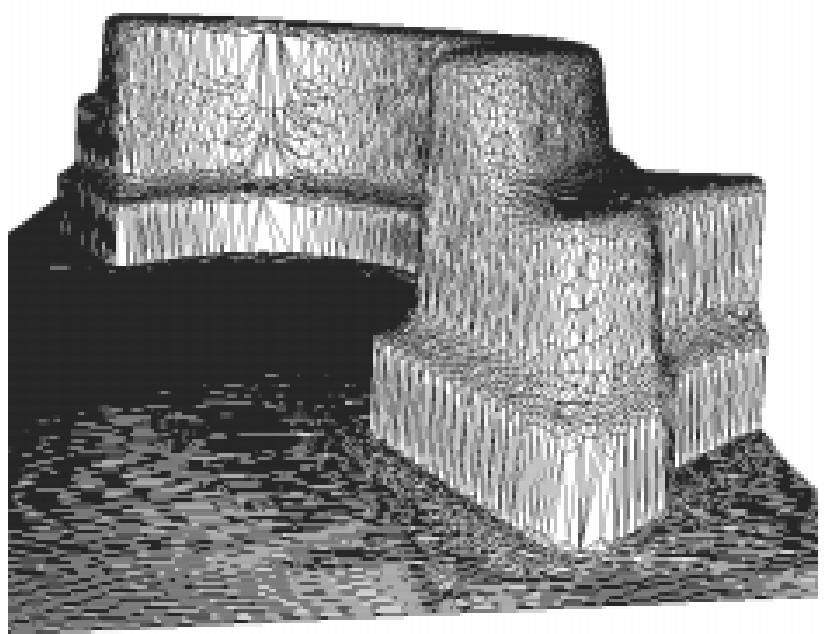

Fig. 7: Connecting rod: deterministic triangulation.

It may seem that the EA profits from the better quality criterion only. But today, there is no deterministic method using the TAC which converges to the optimum. Since determistic methods accept improvements only, they always find a local optimum close to the initial configuration. Moreover, one of the major advantages of the EA over specialized algorithms is its ability to use the best criterion available.

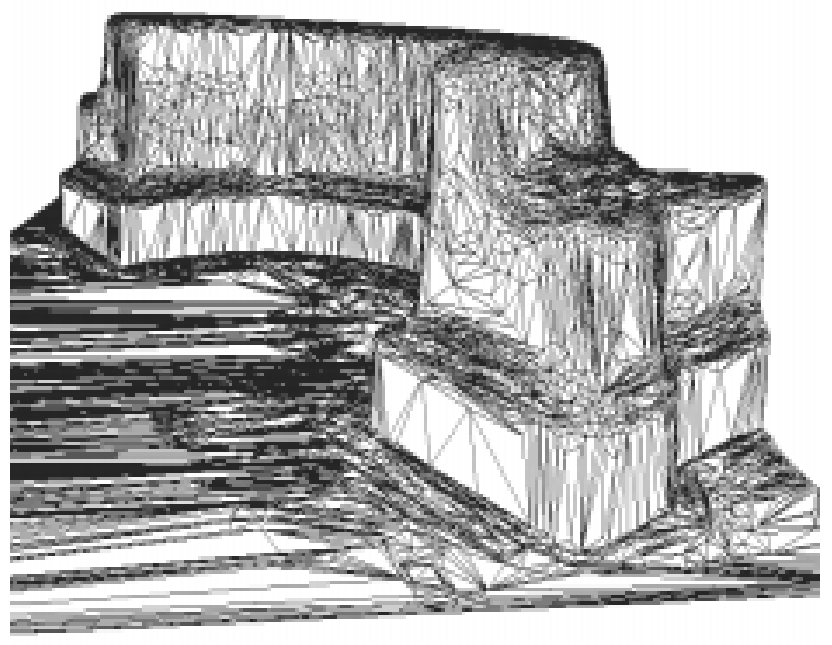

Fig. 8: Connecting rod: EA triangulation. 


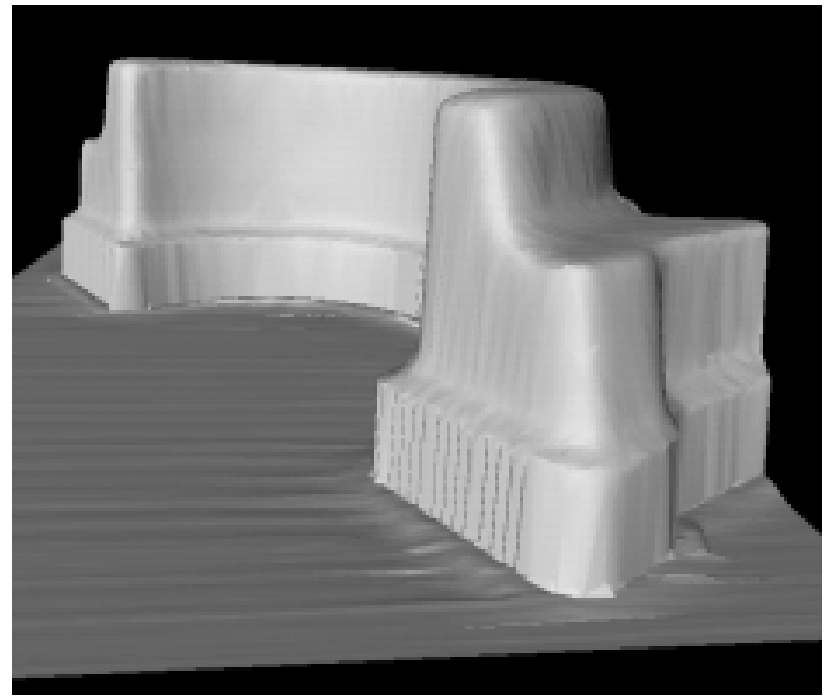

Fig. 9: Connecting rod: surface reconstructed from the deterministic triangulation.

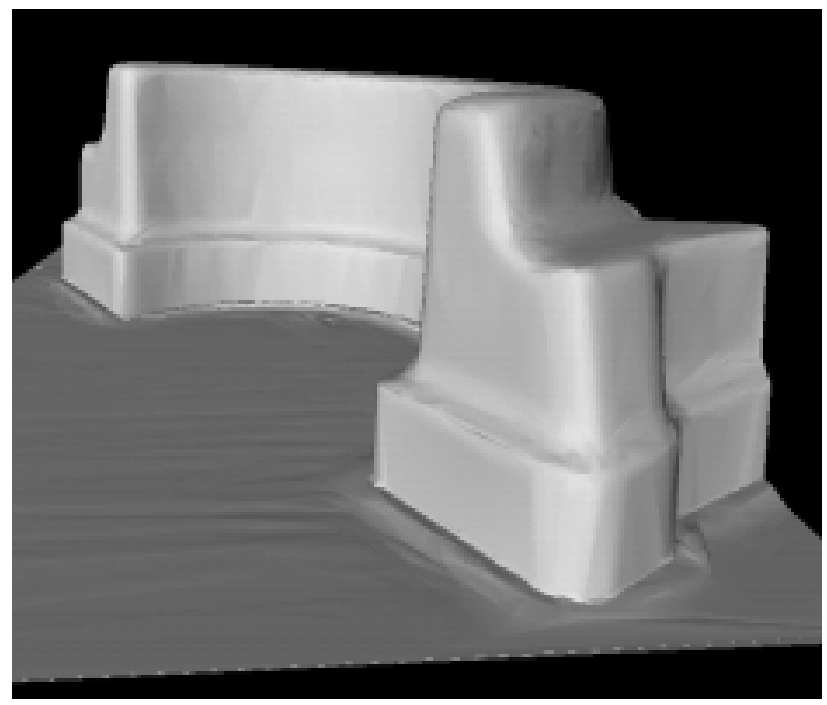

Fig. 10: Connecting rod: surface reconstructed from the EA triangulation.

\section{Conclusions}

This comparison has shown that the EA triangulation is superior to traditional deterministic algorithms, and that the EA is at least an alternative to the SA. Although the SA produces results of the same quality in shorter time, the EA may be preferable for large problem instances due to the existence of efficient parallel implementations. And from a more general view, the SA and EA are probabilistic search methods which could be easily modeled in a common framework.

The successful application of EAs to surface reconstruction presented here can be seen as a first feasibility study. It is very likely that both the performance of the algorithm and the quality of the results can be further improved by advanced operators, such as recombination and selfadaptive mutation rates.

\section{Acknowledgments}

Joachim Sprave gratefully acknowledge support by the BMBF, grant 01 JR 509 A.

\section{References}

[1] L. Alboul and R. van Damme. Polyhedral Metrics in Surface reconstruction: Tight Triangulations. University of Twente, Dep. of Applied Mathematics, Technical Report, 1995.

[2] Th. Bäck. Evolutionary Algorithms in Theory and Practice. Oxford University Press, New York, 1996.

[3] N. Dyn, D. Levin, and S. Rippa. Data Dependent Triangulations for Piecewise Linear Interpolation. pages 137-154. IMA Journal of Numerical Analysis 10, 1990.

[4] S. Kirkpatrick, C. D. Gelatt, and M. P. Vecchi. Optimization by simulated annealing. Science, 220:671$680,1983$.

[5] Z. Michalewicz. Genetic Algorithmus + Data Structures $=$ Evolution Programms. Springer-Verlag Berlin Heidelberg, 3. revised and extended edition, 1996.

[6] G. Prestifilippo. Optimale Triangulierung durch Anwendung von deterministischen und Evolutionären Algorithmen. Diploma thesis, Unversity of Dortmund, 1997.

[7] L. L. Schumaker. Computing optimal triangulations using simulated annealing. pages 329-345. Computer Aided Geometric Design 10 (30), 1993. 
[8] L. L. Schumaker and E. Quak. Cubic spline fitting using data dependent triangulations. pages 293-301. Computer Aided Geometric Design 7, North-Holland, 1990. 